\title{
Expansion of HIV-specific T follicular helper cells in chronic HIV infection
}

\author{
Madelene Lindqvist, ${ }^{1}$ Jan van Lunzen, ${ }^{2}$ Damien Z. Soghoian, ${ }^{1}$ Bjorn D. Kuhl, ${ }^{1}$ \\ Srinika Ranasinghe, ${ }^{1}$ Gregory Kranias, ${ }^{1}$ Michael D. Flanders, ${ }^{1}$ Samuel Cutler, ${ }^{1}$ Naomi Yudanin, ${ }^{3}$ \\ Matthias I. Muller, ${ }^{1}$ Isaiah Davis, ${ }^{1}$ Donna Farber, ${ }^{3}$ Philip Hartjen, ${ }^{2}$ Friedrich Haag, ${ }^{4}$ \\ Galit Alter, ${ }^{1}$ Julian Schulze zur Wiesch, ${ }^{2}$ and Hendrik Streeck ${ }^{1}$
}

\begin{abstract}
${ }^{1}$ Ragon Institute of MGH, MIT, and Harvard Massachusetts General Hospital, Harvard Medical School, Boston, Massachusetts, USA. ${ }^{2}$ Infectious Diseases Unit, University Medical Center Eppendorf, Hamburg, Germany. ${ }^{3}$ Columbia Center for Translational Immunology, Columbia University, New York, New York, USA. ${ }^{4}$ Department of Immunology, University Medical Center Eppendorf, Hamburg, Germany.
\end{abstract}

\begin{abstract}
HIV targets CD4 T cells, which are required for the induction of high-affinity antibody responses and the formation of long-lived B cell memory. The depletion of antigen-specific CD4 T cells during HIV infection is therefore believed to impede the development of protective B cell immunity. Although several different HIVrelated $B$ cell dysfunctions have been described, the role of CD4 $\mathrm{T}$ follicular helper (TFH) cells in HIV infection remains unknown. Here, we assessed HIV-specific TFH responses in the lymph nodes of treatment-naive and antiretroviral-treated HIV-infected individuals. Strikingly, both the bulk TFH and HIV-specific TFH cell populations were significantly expanded in chronic HIV infection and were highly associated with viremia. In particular, GAG-specific TFH cells were detected at significantly higher levels in the lymph nodes compared with those of GP120-specific TFH cells and showed preferential secretion of the helper cytokine IL-21. In addition, TFH cell expansion was associated with an increase of germinal center B cells and plasma cells as well as IgG1 hypersecretion. Thus, our study suggests that high levels of HIV viremia drive the expansion of TFH cells, which in turn leads to perturbations of $B$ cell differentiation, resulting in dysregulated antibody production.
\end{abstract}

\section{Introduction}

One of the hallmarks of HIV infection is a progressive dysfunction of the humoral immune response (1). Even during the earliest stages of HIV infection, a skewing of the functional and phenotypic properties of B cells can be observed that is only partially restored following the introduction of long-term antiretroviral treatment (2). While the majority of peripheral B cells in healthy individuals are either resting naive or memory B cells, several additional subpopulations emerge during the chronic phase of HIV infection that are normally present at negligible levels (1). In particular, immature and pregerminal center B cells as well as plasmablasts have been shown to be significantly expanded in HIV-infected individuals (1, 3). In addition, persistent activation of immature B cell populations during chronic infection appears to drive the secretion of high levels of nonspecific polyclonal antibodies, resulting in hypergammaglobulinemia $(4,5)$.

Several mechanisms have been suggested to account for these B cell abnormalities observed during HIV infection. While B cells themselves are not targets for HIV, viral replication and persistently high levels of viral proteins have been shown to be associated with nonspecific $B$ cell activation as well as deficiencies in the formation of $B$ cell memory (6-8). Indeed, both dysfunctions are partially resolved following initiation of antiretroviral treatment, with subsequent reduction of viremia $(1,5,9,10)$. It has been suggested that the massive infection and depletion of CD4 T cells that occurs during HIV infection in all compartments of the body (11) may play a pivotal role in the profound skewing of the B cell compartment. The inter-

Authorship note: Madelene Lindqvist, Jan van Lunzen, and Hendrik Streeck contributed equally to this work.

Conflict of interest: The authors have declared that no conflict of interest exists. Citation for this article: J Clin Invest. 2012;122(9):3271-3280. doi:10.1172/JCI64314. action of B cells and CD4 T cells has been shown to be fundamental for the development of high-affinity matured antibodies and for the development of long-lived memory B cells (12). A highly specialized CD4 helper subset within the lymphoid follicle, the $\mathrm{T}$ follicular helper (TFH) cells, has been shown to undergo a tight interaction with antigen-specific B cells that induces antibody affinity maturation as well as the development of memory B cells and plasma cells (13). TFH cells have been suggested to be a separate lineage of CD4 T cell, characterized by high-level surface expression of the lymphoid follicle homing receptor CXCR5 and programmed death-1 (PD-1) as well as the transcription factor B cell lymphoma 6 (BCL6) (14). Furthermore, TFH cells have also been shown to preferentially secrete the cytokine IL-21 that is pivotal for germinal center formation, germinal center B cell proliferation, and B cell maturation (13). During this process, germinal center B cells present cognate peptide to TFH cells and, in turn, receive survival signals that lead to further differentiation into memory B cells and plasma cells. Under normal conditions, TFH cells, in addition to follicular dendritic cells, rescue only the highest-affinity germinal center B cells for survival and further development, and it has been suggested that the presence of TFH cells appears to be the limiting step in this process (12). However, it is not known how this cellular subset is affected by HIV infection. Moreover, in HIV infection, nonspecific B cell proliferation is widespread, and low-affinity B cells mature inappropriately into plasma cells (4). Yet, the mechanism behind this TFH-mediated alteration in the $\mathrm{B}$ cell subsets and accumulation of plasma cells is not immediately apparent: memory CD4 T cells are preferentially depleted during HIV infection, which is initially at odds with the requirement of TFH cells for plasma cell differentiation. We therefore sought to investigate the role of TFH cells in chronic HIV infection and to determine the mechanism by which these cells may contribute to B cell dysfunction and the development of hypergammaglobulinemia. 
A

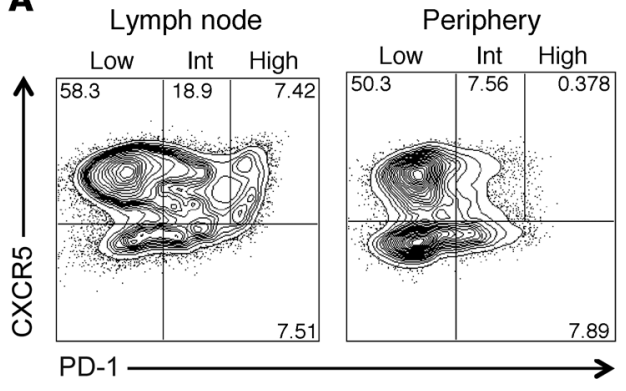

B

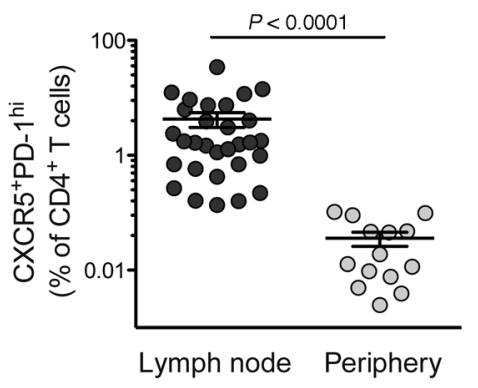

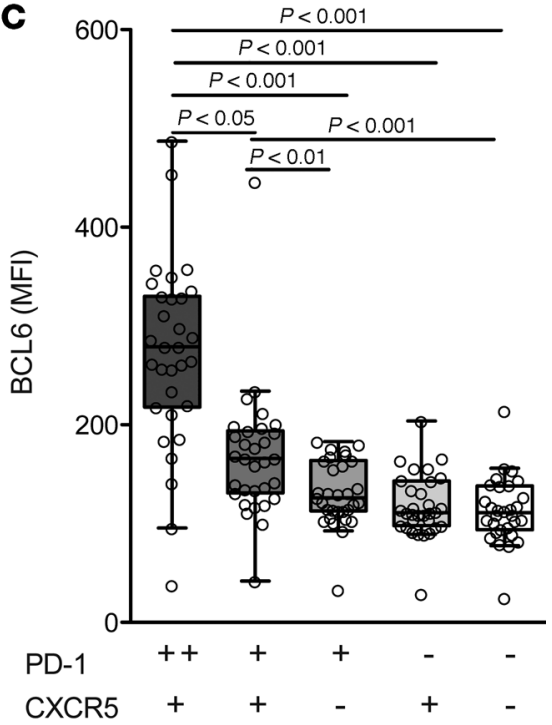

Figure 1

High frequency of CXCR5+PD-1 $1^{\text {hi }}$ CD4 T cells with BCL6 expression in human lymph nodes. (A) Representative plots of CXCR5 and PD-1 expression on CD4 T cells in lymph nodes and corresponding PBMCs of HIV-infected individuals. Numbers represent fraction (\%) of total CD4 T cells. (B) The frequency of CXCR5 $5^{+P D}-1^{\text {hi }}$ cells of CD4 T cells is significantly higher in lymph nodes (average, $4.3 \% \pm 7 \%$ ) compared with periphery (average, $0.04 \% \pm 0.04 \%)(P<0.0001)$. Symbols represent individual samples; horizontal bars represent mean; and error bars show SEM. (C) Median fluorescence intensity of BCL6 expression based on CXCR5 or PD-1 expression in different CD4 T cell subsets in the lymph node. The highest BCL6 expression was detected within the CXCR5+PD-1 hi CD4 T cell subset $(n=31)$. In box-and-whisker plots, horizontal bars indicate the medians, boxes indicate 25th to 75 th percentiles, and whiskers represent 1.5 times the interquartile distance (Tukey). B and C include all HIV-infected and uninfected individuals $(n=31)$.

\section{Results}

Human TFH cells are characterized as $C X C R 5^{+} P D-1^{\text {hi }} \mathrm{CD} 4 \mathrm{~T}$ cells within the lymph nodes. To determine the role of virus-specific TFH cells in chronic HIV infection, we first defined the characteristics of TFH cells in human lymph nodes. Sixteen axillary lymph nodes were surgically resected from chronically HIV-infected individuals with viral loads averaging 121,400 HIV RNA copies/ml $( \pm 47,235$ HIV RNA copies/ml) and $10 \mathrm{HIV}$-infected subjects on long-term antiretroviral therapy (ART) with fully suppressed viremia $(<50$ HIV RNA copies/ml). Parallel blood draws were obtained from 14 individuals. In addition, we received axillary lymph nodes from 7 HIV-negative individuals.

As previous studies in mice and human tonsils have suggested that the expression of CXCR5 and PD-1 well characterizes TFH cells (14-17), we first confirmed the expression of both receptors on CD4 T cells from human lymph nodes in comparison to that on peripheral CD4 T cells. We were able to detect a CXCR5 ${ }^{+} \mathrm{PD}-1^{+} \mathrm{CD} 4 \mathrm{~T}$ cell population in both lymph nodes and blood, but observed a distinct population of CXCR5 ${ }^{+}$PD-1 ${ }^{\text {hi }}$ CD4 $\mathrm{T}$ cells in the lymph nodes that was almost completely absent in the periphery (Figure 1A). Interestingly, the frequency of this population was about 100-fold higher in the lymphoid compartment compared with that in the matched peripheral samples (average, $4.3 \% \pm 7 \%$ vs. $0.04 \% \pm 0.04 \%, P<0.0001$; Figure $1 \mathrm{~B})$, but we did not observe an association between the frequencies of CD4 T cells with TFH characteristics in the periphery (data not shown). To further verify the lineage commitment of the CXCR5 ${ }^{+}$ $\mathrm{PD}-1^{\text {hi }} \mathrm{CD} 4 \mathrm{~T}$ cell population, we next determined the expression of the previously described TFH transcription factor BCL6 (18), which was most highly expressed in the $\mathrm{CXCR}^{+} \mathrm{PD}-1^{\text {hi }} \mathrm{CD} 4 \mathrm{~T}$ cell population, as previously described $(P<0.001$; Figure $1 \mathrm{C}$ and refs. 14,15$)$.
We next determined the functional characteristics of different CD4 $\mathrm{T}$ cell populations in the lymph nodes after nonspecific stimulation with PMA and ionomycin. IL-21 has been previously suggested to be one of the cardinal cytokines of TFH cells and pivotal for the germinal center reaction during $B$ cell differentiation (19). Indeed, we found the highest levels of IL-21 expression in the $\mathrm{CXCR} 5^{+} \mathrm{PD}-1^{\text {hi }} \mathrm{CD} 4 \mathrm{~T}$ cell population that was significantly higher, even compared with the overall CD4 $\mathrm{T}$ cell responses in the periphery $(P<0.001$; Figure 2 , A and $B)$. We also observed a higher frequency of TNF- $\alpha-$ and IFN- $\gamma-$ secreting cells within the CXCR5 ${ }^{+} \mathrm{PD}-1^{\text {hi }}$ population that were likewise more abundant in the lymph node than in the periphery $(P<0.01$ and $P<0.001$, respectively). We next determined the memory phenotype of the 3 different CXCR5- and PD-1expressing CD4 $\mathrm{T}$ cell populations and found that the TFH cells, defined as $\mathrm{CXCR}^{+} \mathrm{PD}-1^{\text {hi }} \mathrm{CD} 4 \mathrm{~T}$ cells, were predominantly effector memory CD4 T cells (CCR7-CD45RA-), while the $\mathrm{CXCR}^{+} \mathrm{PD}-1^{\text {int }}$ and $\mathrm{CXCR} 5^{+} \mathrm{PD}-1^{\text {lo }}$ population were enriched for central memory CD4 T cells (CCR7 $\left.{ }^{+} \mathrm{CD} 45 \mathrm{RA}^{-}\right)$(Figure 2C). Thus, these findings confirm a similar phenotype and expression pattern of CD4 TFH cell populations in human lymph nodes, as previously described in mice.

Expansion of TFH cells during chronic HIV infection. As HIV infection is marked by a massive depletion of activated CD4 memory $\mathrm{T}$ cells in all compartments of the body (11), it has been speculated that this is a major contributing factor to the profound impairment of the B cell responses (20-22). However, the effects of HIV infection on the TFH cell subset are still unknown. We therefore assessed the frequency of TFH (CXCR5 ${ }^{+}$PD-1 $\left.{ }^{\text {hi }}\right)$ CD4 $\mathrm{T}$ cells in the lymph nodes of chronically infected untreated 
A

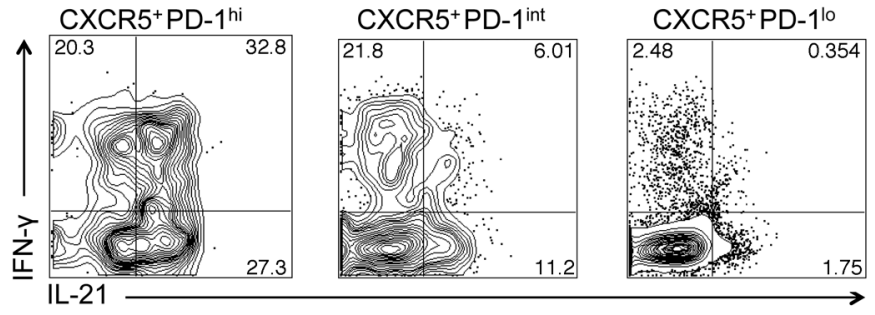

B

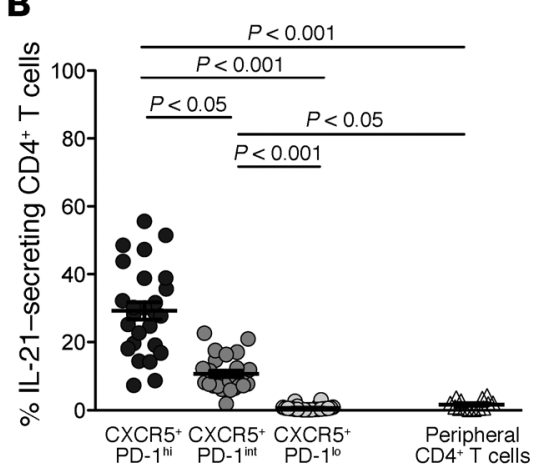

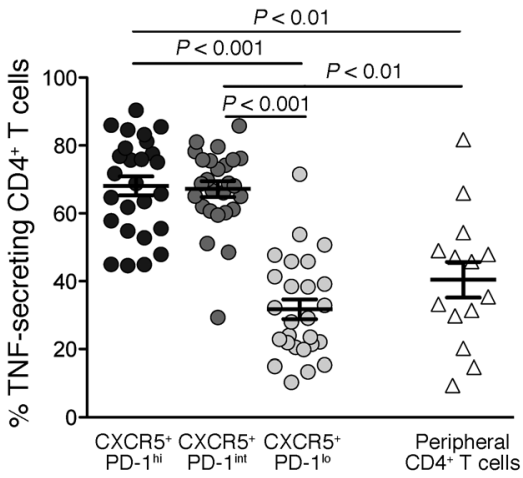

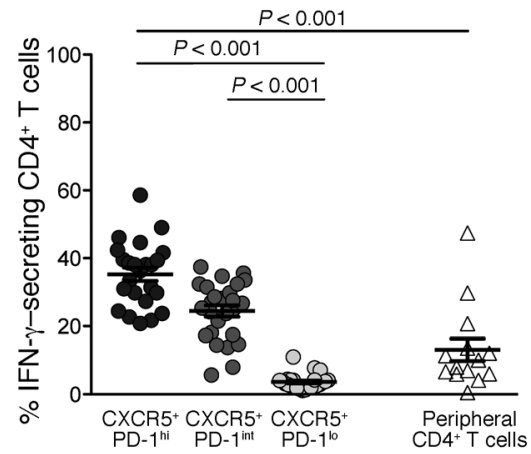

C

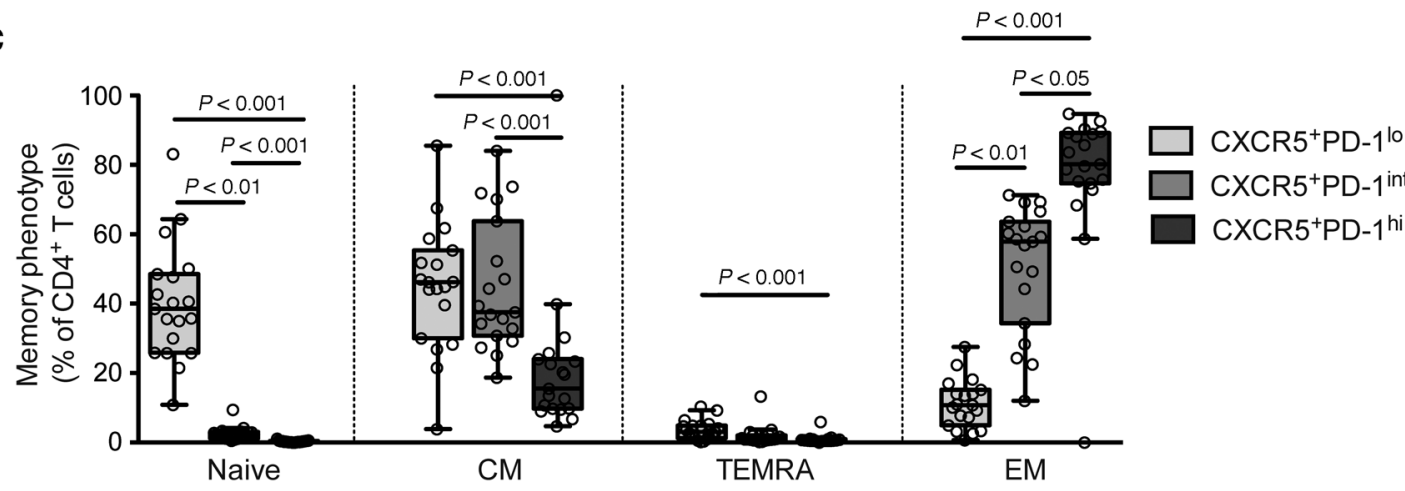

Figure 2

TFH cells in the lymph node are predominantly effector memory cells and secrete high levels of IL-21. (A) Representative flow plots of IFN- $\gamma$ and IL-21 production of TFH cells in the lymph node. Numbers represent fraction (\%) of total CD4 T cells. (B) Frequency of cytokine production dependent on CXCR5 and PD-1 expression in CD4 T cell subsets in the lymph node and periphery. Symbols represent individual samples; horizontal bars represent mean; and error bars show SEM. (C) Definition of memory phenotype of CD4 T cell subsets in the lymph node demonstrated the

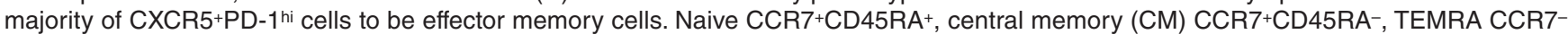
$\mathrm{CD}^{2} 5 \mathrm{RA}^{+}$, and effector memory (EM) CCR7-CD45RA- $(n=19)$ cells are shown. In box-and-whisker plots, horizontal bars indicate the medians, boxes indicate 25th to 75 th percentiles, and whiskers represent 1.5 times the interquartile distance (Tukey).

and long-term ART-treated patients compared with that in HIV-seronegative individuals (Figure 3A). Surprisingly, we found significantly increased frequencies of TFH cells in HIVinfected individuals compared with those in uninfected subjects (average, $5.0 \% \pm 1.42 \%$ vs. $0.51 \% \pm 0.24 \% ; P=0.003$ ), and this association was also reflected with increased BCL6 expression within the TFH CD4 cell population $(P<0.01$ and $P=0.01$, respectively; Figure 3, B and C). While TFH cell frequency was 2 -fold higher in patients with high viremia compared with that in those subjects successfully suppressed on long-term ART, we did not observe a direct correlation between viral loads and TFH cell frequency, either in the periphery or lymph nodes, suggesting that additional factors might influence the expansion of TFH cells (data not shown).
We next assessed whether the increase in overall TFH cells in HIV-infected subjects might also be reflected in an antigen-specific TFH cell expansion or rather is independent of its specificity. To assess the HIV-specificity of TFH cells, we stimulated cells extracted from lymph nodes of HIV-infected subjects with overlapping peptide pools corresponding to the clade $\mathrm{B}$ envelope protein GP120 or the core protein GAG. HIV-specific TFH responses were readily detectable in all HIV-infected individuals (Figure 3, D and E). Surprisingly, we observed that the GAG-specific TFH responses were, on average, 5 times stronger than the TFH responses against envelope GP120 $(P<0.0001)$. Previous studies have demonstrated a strong linkage and dependency between the protein specificity of CD4 $\mathrm{T}$ cell and antibody responses, suggesting that CD4 $\mathrm{T}$ cell help for antibody development is dependent on tandem 
A

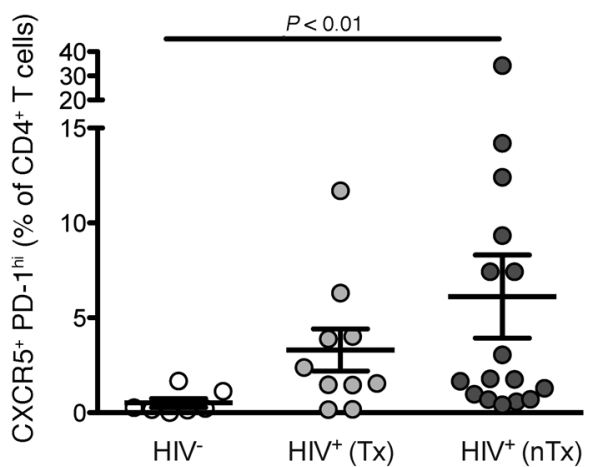

B

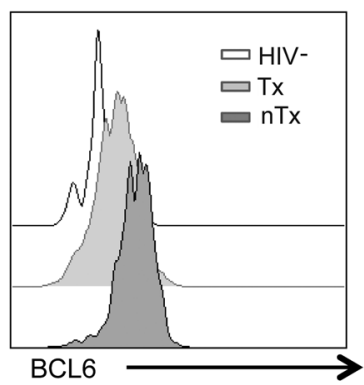

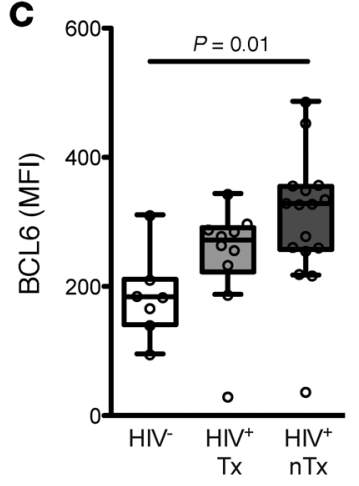

D

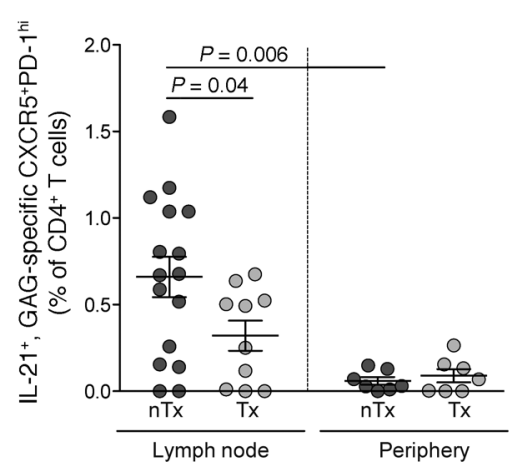

$\mathbf{E}$

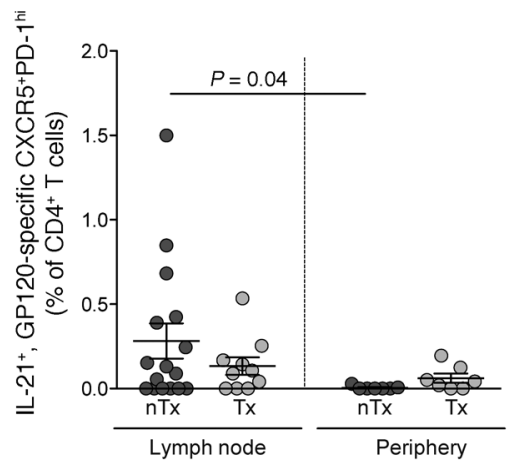

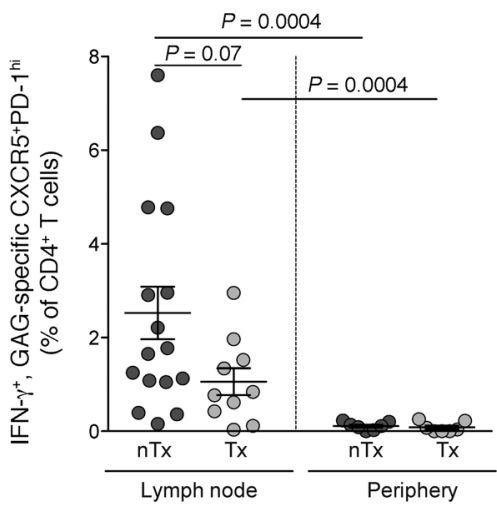
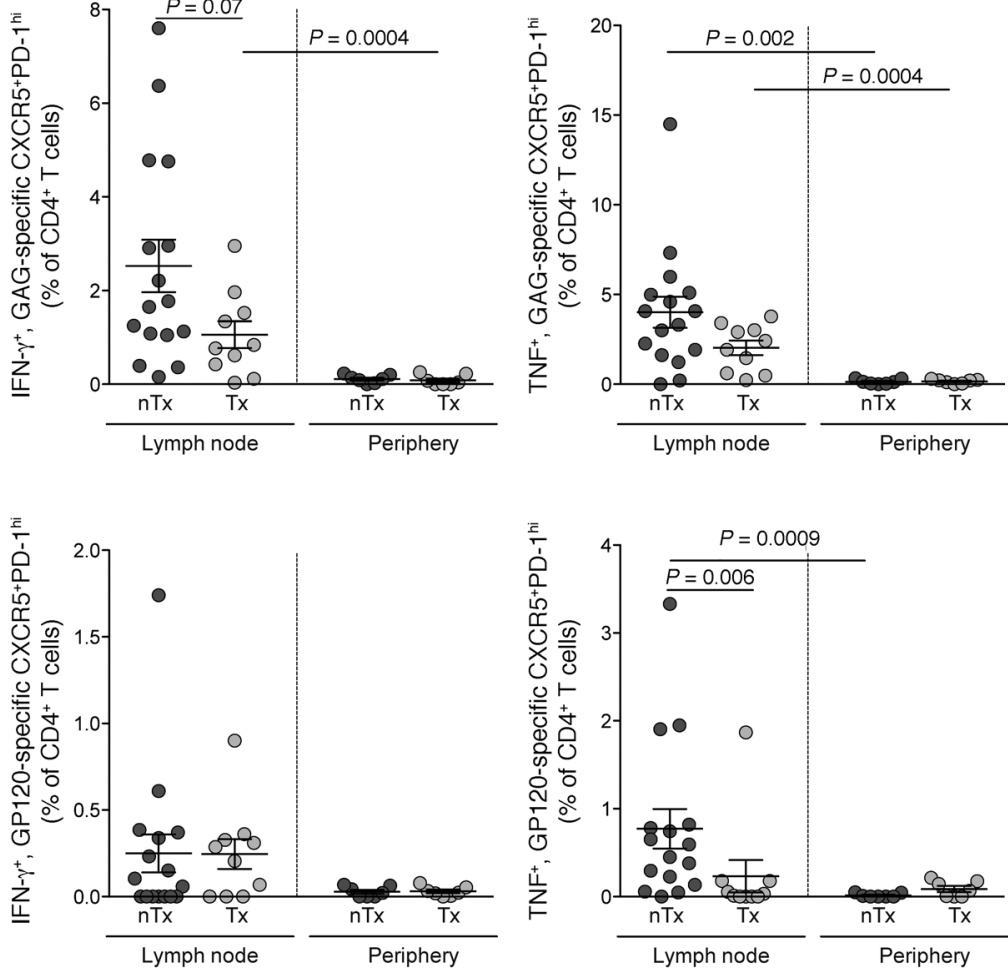

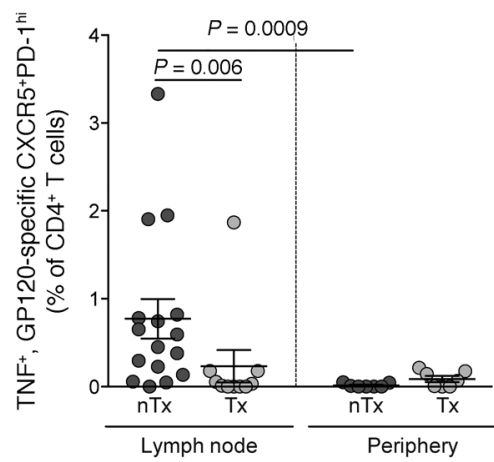

Figure 3

Expansion of HIV-specific TFH cells in chronic infection. Mononuclear cells from lymph nodes were stimulated with overlapping peptide pools for HIV-1 GAG and GP120 for 6 hours and subsequently examined by intracellular multiparameter flow cytometry. (A) Significantly higher frequencies of CXCR5+PD-1 hi CD4 T cells can be detected in chronic HIV-positive (treatment-naive [nTx]) individuals $(6.1 \% \pm 8.7 \%)$ compared with those in ART-treated (Tx) $(3.3 \% \pm 3.5 \%)$ and HIV-negative $(0.5 \% \pm 0.6 \%)$ individuals. (B and C) Significantly higher BCL6 expression was detected in CXCR5+PD- $1^{\text {hi }}$ CD4 T cells in chronic HIV-positive individuals compared with that in uninfected individuals, as shown by representative histogram plot and median fluorescence intensity level, respectively $(P<0.01)$. In box-and-whisker plots, horizontal bars indicate the medians, boxes indicate 25th to 75th percentiles, and whiskers represent 1.5 times the interquartile distance (Tukey). (D and E) Higher frequency of GAG- and GP120-specific cytokine responses, respectively, in CXCR5+PD-1 hi CD4 T cells in lymph nodes of treatment-naive individuals compared with that in individuals on ART, and the overall CD4 T cell responses in periphery. (A, D, and E) Symbols represent individual samples; horizontal bars represent mean; and error bars show SEM.

recognition of the same protein (23). While GAG-specific antibody responses have been described to emerge early in infection, they decline with disease progression and are often even absent in the end stage of chronic infection (reviewed in ref. 24 and refs. 25-28). In contrast, broadly neutralizing antibodies against structures in envelope emerge in the chronic phase of infection. Thus, the dominance of GAG-specific CD4 $\mathrm{T}$ cell responses in the setting of
TFH cells during chronic infection is surprising, and it remains to be determined what role GAG-specific TFH responses play in the lymph nodes of HIV-infected patients.

Similar to the expansion of total frequency of TFH cells, we also observed that the overall frequency of HIV-specific TFH cells was higher in chronic HIV-infected individuals compared with that in treated patients. The HIV-specific TFH cells secreted high levels of 
A

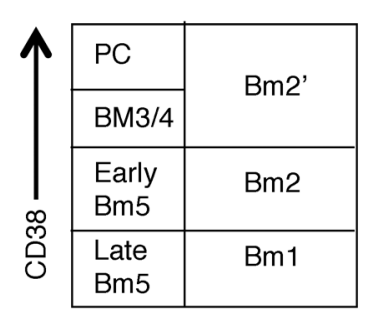

$\lg \mathrm{D}$
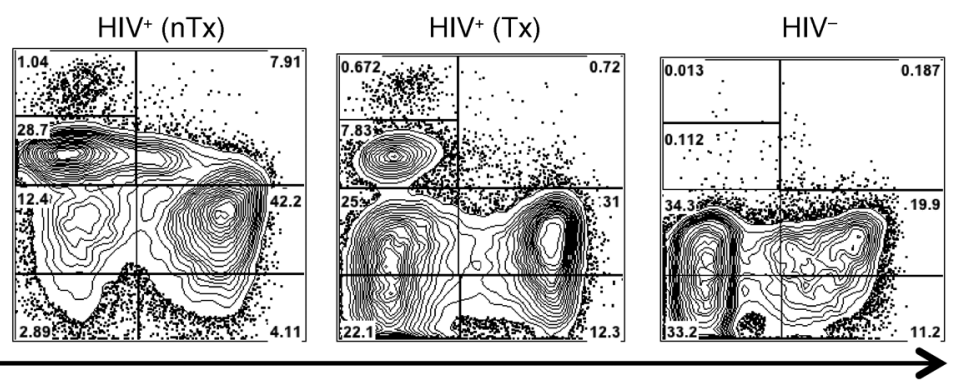

B
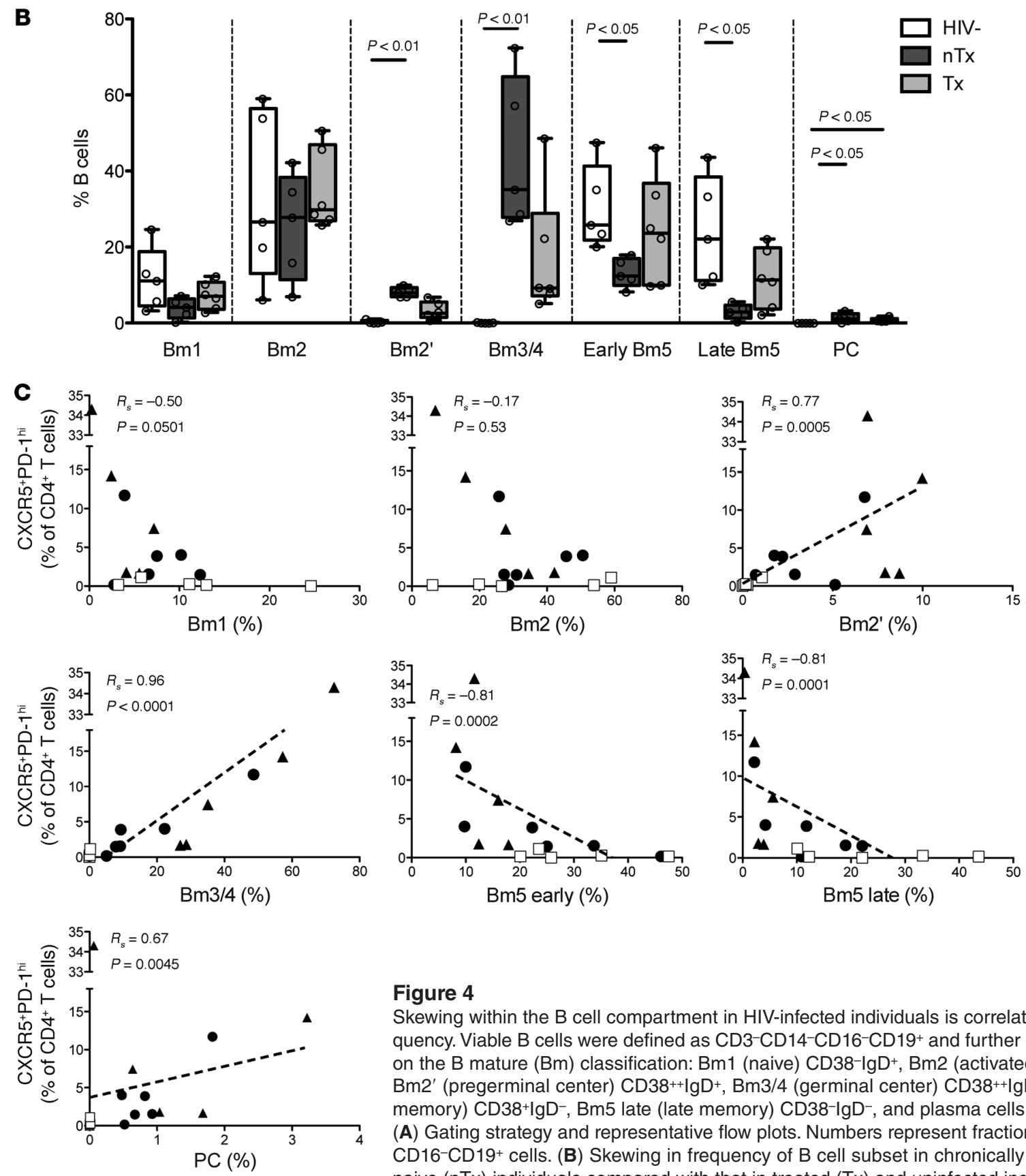

Figure 4

Skewing within the B cell compartment in HIV-infected individuals is correlated with TFH cell frequency. Viable B cells were defined as CD3-CD14-CD16-CD19+ and further characterized based on the $\mathrm{B}$ mature $(\mathrm{Bm})$ classification: $\mathrm{Bm} 1$ (naive) $\mathrm{CD}^{-} 8^{-} \mathrm{IgD}{ }^{+}, \mathrm{Bm} 2$ (activated naive) $\mathrm{CD}^{2} 8^{+} \lg \mathrm{D}^{+}$, Bm2' (pregerminal center) CD38++lgD+, Bm3/4 (germinal center) CD38++lgD-, Bm5 early (early memory) CD38 ${ }^{+} \mathrm{IgD}{ }^{-}, \mathrm{Bm} 5$ late (late memory) CD38-lgD-, and plasma cells (PCs) CD38 ${ }^{+++}$IgD ${ }^{-}$. (A) Gating strategy and representative flow plots. Numbers represent fraction (\%) of CD3-CD14CD16-CD19+ cells. (B) Skewing in frequency of B cell subset in chronically infected, treatmentnaive $(\mathrm{nTx})$ individuals compared with that in treated $(\mathrm{Tx})$ and uninfected individuals. In box-andwhisker plots, horizontal bars indicate the medians, boxes indicate 25th to 75th percentiles, and whiskers represent 1.5 times the interquartile distance (Tukey). (C) Significant correlations between $B$ cell subsets and TFH cell frequency in the lymph nodes of uninfected (white squares) and HIVinfected, untreated (black triangles) and treated (black circles) individuals. 

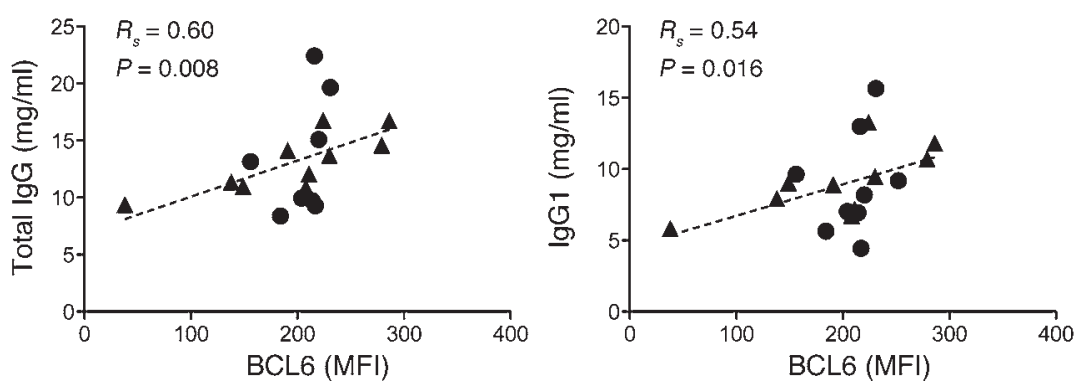

\begin{abstract}
Figure 5
Hypergammaglobulinemia is associated with BCL6 expression in TFH cells. Significant correlation between the expression of BCL6 in TFH cells and levels of total serum IgG and IgG1 antibody levels in HIV-infected untreated (black triangles) and treated (black circles) individuals.
\end{abstract}

IL-2 1 in addition to IFN- $\gamma$ and TNF- $\alpha$ (Figure 3, D and E, respectively), and about one-quarter of the HIV-specific TFH cells produced more than one cytokine (data not shown). Interestingly, the frequency of HIV-specific TFH cells was on average 20-fold higher in the lymph nodes compared with the overall HIV-specific CD4 $\mathrm{T}$ cell response detected in the periphery (average, $1.09 \% \pm 0.15 \%$ vs. $0.05 \% \pm 0.02 \%$, respectively; $P<0.0001)$. Taken together, our data demonstrate a significant expansion of HIV-specific TFH CD4 $\mathrm{T}$ cells in chronically HIV-infected individuals that is associated with HIV viremia and skewed toward the GAG protein.

Expansion of TFH cells in HIV infection is associated with Ig G gammaglobulinemia and skewing of the $B$ cell phenotype. Despite the reduction in CD4 T cell numbers, chronic HIV is generally accompanied by hypergammaglobulinemia in the blood serum $(4,29,30)$. The reason for this condition is still unknown. As we observed a significant expansion in the frequency of TFH cells that are both functionally active and HIV specific, we reasoned that the expansion - rather than its loss - might drive these changes in the $\mathrm{B}$ cell compartment. We therefore assessed the B cell maturation phenotype in the lymph nodes of HIV-positive and -negative individuals and distinguished $7 \mathrm{~B}$ cell populations based on the differential expression of CD38 and IgD (Figure 4, A and B). We referred to the populations according to the "B mature" Bm1$\mathrm{Bm} 5$ nomenclature previously established for the development status of $\mathrm{B}$ cells from naive $(\mathrm{Bm} 1$ and $\mathrm{Bm} 2)$ through pregerminal center $\left(B m 2^{\prime}\right)$ and germinal center $(\mathrm{Bm} 3 / 4)$ to memory cells (Bm5) and plasma cells $(31,32)$. Interestingly, we observed a significant skewing of the $\mathrm{B}$ cell population toward germinal center $\left(\mathrm{Bm}^{\prime}\right)$ and $(\mathrm{Bm} 3 / 4) \mathrm{B}$ cells in chronically HIV-infected individuals compared with that in HIV-negative individuals. This observed skewing was still modestly greater when comparing chronically infected individuals with those on long-term ART (Figure 4B). We also detected an increase of immature transitional B cells in untreated HIV-infected individuals compared with that in uninfected subjects (average, $4.9 \% \pm 0.46 \%$ vs. $0.26 \% \pm 0.16 \%$, respectively; $P<0.01$ ) (Supplemental Figure 3; supplemental material available online with this article; doi:10.1172/JCI64314DS1). Moreover, we observed a significant decrease in the frequency of memory B cells in chronically infected individuals compared with that in uninfected individuals $(13.21 \% \pm 1.71 \%$ vs. $30.4 \% \pm 4.95 \%$, respectively; $P<0.05)$. The loss of memory B cells was accompanied by an increase of plasma cells in both treatment-naive $(1.33 \%$ $\pm 0.54 \%)$ and treated individuals $(0.87 \% \pm 0.20 \%)$ compared with that in uninfected individuals $(0.006 \% \pm 0.002 \% ; P<0.05)$, suggesting a link between the perturbation of $\mathrm{B}$ cell phenotypes and the expansion of TFH cells. Indeed, we observed a strong positive correlation between the expansion of both $\mathrm{Bm} 2^{\prime}\left(\mathrm{r}_{\mathrm{s}}=0.77\right.$; $P=0.0005)$ and $\mathrm{Bm} 3 / 4\left(\mathrm{r}_{\mathrm{s}}=0.96 ; P<0.0001\right)$ germinal center $\mathrm{B}$ cells and lymphoid plasma cells $\left(\mathrm{r}_{\mathrm{s}}=0.67 ; P=0.0045\right)$ as well as loss of memory B cells $\left(r_{s}=-0.81, P=0.0002\right)$ and the frequency of TFH cells irrespective of their infection status (Figure 4C). Similarly, this correlation was also detected between immature transitional B cells and the frequency of TFH cells $\left(r_{\mathrm{s}}=0.78 ; P=0.0009\right)$ (Supplemental Figure 3).

Previous reports have demonstrated that the differentiation into plasma cells as well as germinal center B cells is a TFH-dependent process and that, in fact, a large proportion of TFH functions are dedicated to B cell survival and proliferation (13). However, while it is possible that the expansion of TFH cells might be an underlying factor for the skewing observed in the B cell phenotype, we did not observe any associations in HIV-negative individuals. Thus, it is plausible that an underlying defect or dysregulation within the TFH cells in HIV infection is responsible for the observed perturbation in the B cell compartment.

Given the significant changes in the B cell compartment, we next determined whether the expansion of TFH cells is also associated with hypergammaglobulinemia. We therefore assessed the IgG antibody levels in the serum of the HIV-infected individuals and found a trend between the expansion of TFH cells and IgG $(P=0.09$; data not shown). Interestingly, we detected a significant correlation between the BCL6 expression in TFH cells and the total IgG antibody concentration in HIV-infected, untreated, and treated individuals $\left(\mathrm{r}_{\mathrm{s}}=0.60 ; P=0.008\right.$; Figure 5$)$. This association was mainly driven by the IgG subclass IgG1, which constituted $70 \%$ of the secreted IgG antibodies $\left(\mathrm{r}_{\mathrm{s}}=0.65, P=0.016\right)$. However, no association was detected between the level of BCL6 in TFH cells with IgG2, IgG3, or IgG4 subclasses (data not shown) and any HIV-specific TFH cell responses.

Taken together, our data indicate that the expansion of TFH cells in chronic HIV infection shows substantial association with the skewing of the B cell compartment. Moreover, HIV-induced increase in the expression of BCL6 in TFH cells was also significantly associated with hypersecretion of IgG1 antibodies.

\section{Discussion}

One of the fundamental characteristics of HIV pathogenesis is a widespread perturbation of the B cell compartment, yet the mechanisms underlying this dysfunction remain only poorly understood (1). In this work, we examined the role of human TFH cells in the lymph nodes of healthy controls and individuals at different stages of HIV infection and observed a viral load-dependent expansion of the frequency of TFH cells and of BCL6 expression within TFH cells. Moreover, this expansion of TFH cells during chronic HIV infection was associated with a profound skewing of the B cell compartment toward germinal center B cells and plasma cells and a corresponding reduction in the memory B cell popula- 
tion in the lymph nodes. In addition, the expansion of TFH cells was also significantly associated with hypergammaglobulinemia that was mainly driven by an accruement of IgG1.

During HIV infection a massive depletion of activated memory CD4 T cells occurs throughout all compartments of the body (11). Unexpectedly, however, we observed a relative expansion - and not decrease - of TFH cells to the overall CD4 T cell count in the lymph nodes of all HIV-infected patients. While it remains to be determined whether this expansion is also reflected as an increase in the absolute number of TFH cells, it was also seen in the HIVspecific TFH cell compartment and was highest in subjects with untreated HIV infection. However, we did not observe a direct correlation between individual peripheral viral loads and lymph node TFH cell frequency - rather, the presence of viremia in general was associated with higher TFH levels. Similar observations have been made in LCMV infection: in an elegant study by Fahey et al., the authors demonstrate that during viral persistence, CD4 T cells preferentially differentiate into TFH cells (33).

Studies from the mouse model have demonstrated that the development of TFH cells is driven by high-affinity and prolonged binding of the TCR to its cognate antigen (34). Indeed, this has been confirmed to be a mechanism responsible for the expansion of TFH cells during LCMV infection (33). Similarly, our observations indicated that the expansion of the TFH compartment during HIV infection is characterized by a particularly large increase of TFH cells with HIV specificity, which suggests that the presence of HIV viremia is indeed a driving force behind these changes. Given the findings of previous studies demonstrating a preferential infection and depletion of HIV-specific CD4 cells (35), the observed expansion of HIV-specific TFH cells is rather surprising and raises the question of whether TFH cells may be protected from HIV infection. While we were not able to perform these studies in HIV-infected subjects, Petrovas et al. demonstrate that in chronic SIV infection, TFH cells are equally infected compared with other CD4 subsets (36). Thus, these data suggest that the observed expansion is not due to a lower susceptibility of TFH cells to HIV infection.

We further found that the expansion of TFH cells is highly associated with the observed skewing of the B cell compartment. These changes in the B cell subset are most prominently seen as an expansion of germinal center B cells and plasma cells. However, in light of the particular functions of TFH cells and their expansion found in HIV infection, these alterations might not be very surprising. TFH cells initiate the formation and maintenance of the germinal center through the induction of germinal center $\mathrm{B}$ cell differentiation and proliferation (reviewed in ref. 13). Through pathways that are not yet fully understood, TFH cells induce BCL6 expression in B cells that drives their development into germinal center $\mathrm{B}$ cells and promotes the formation of the nascent germinal center. TFH cells then continue to provide proliferative and survival signals to germinal center B cells, most notably through IL-21 secretion $(37,38)$. Under normal conditions, TFH cells represent the limiting factor in germinal center B cell maturation, and only a limited number of cognate germinal center B cells are stimulated and selected for subsequent rounds of differentiation. This limit may no longer apply in HIV infection, in which TFH cells are abundantly expanded and available to provide maturation signals improperly to germinal center B cells, which would normally be selected against. Interestingly, two recent studies by Linterman et al. and Chung et al. demonstrated that a new subset of $\mathrm{T}$ follicular regulatory ( $\mathrm{Tfr}$ ) cells can be found in lymph nodes and function by suppressing germinal center reactions $(39,40)$. Thus, it remains to be determined whether these cells are involved in the significant expansion of germinal center TFH and B cells and how Tfr cells may contribute to the immunopathologies observed during chronic viral infection.

Indeed, several factors may be responsible for the expansion of HIV-specific TFH cells and germinal center B cells, such as general immune activation with increased IL-6 plasma levels, as suggested by Petrovas et al. (36), lymphopenia-driven IL-7 secretion (41), or direct effects of HIV on B cells $(6,7)$. While it is likely that the underlying factors are multifactorial, our results nonetheless suggest that the profound perturbation in B cell subsets and antibody production may be the result of an increase in the natural TFH immune response. In this model, persistent antigenic stimulation drives an abnormal expansion of TFH cells. However, these TFH cells are highly dysregulated, promoting the expansion of B cell subsets that are normally limited by the presence of TFH cells. In particular, high IL-21 responses have been described as a potent inducer of immunoglobulin secretion and plasma cell differentiation $(42,43)$, both of which we found significantly increased in chronic HIV infection. Thus, dysregulated TFH cells, in concert with other factors, may be important in promoting immunoglobulin secretion and may be one of the underlying mechanisms for hypergammaglobulinemia. Our hypothesis evokes strong interest for further studies of TFH cells in HIV infection. In particular, studying TFH cells in the setting of mucosal immunity in the gut, in which the majority of CD4 T cells are depleted, could further shed light on the role of TFH cells in B cell regulation $(44,45)$.

Despite high levels of TFH cells, the majority of HIV-infected patients do not develop broadly neutralizing antibodies that are able to neutralize an array of different viruses (46). This suggests a defect in the affinity maturation process, as higher levels of affinity maturation have been associated with the breadth of antibody neutralization $(47,48)$. However, the reason for the lack of sufficient somatic hypermutation and affinity maturation of the BCR is unknown. It remains to be seen whether the abundance of HIV-specific TFH cells leads to a premature development of end-stage plasma cells that have not undergone several rounds of somatic hypermutation or whether other elements are responsible for the lack of broadly neutralizing antibody development. Determination of the factors involved in the generation of neutralizing antibody breadth and the associated involvement of TFH cells will be critical in guiding the design of HIV immunogens and vaccination strategies in the future.

\section{Methods}

Study participants. A total of 31 individuals were included in this study, of which 24 were chronic, treatment-naive individuals or patients on ART. In addition, $7 \mathrm{HIV}$-negative individuals were studied (Table 1). Surgically removed axillary lymph nodes and matched PBMCs from HIV-infected individuals were obtained from the University Medical Center Eppendorf. HIV-negative lymph node samples were obtained from organ donors, who tested negative for HIV and had no chronic infections, through National Disease Research Interchange, University Medical Center Eppendorf, and Columbia Center for Translational Immunology.

Stimulation. Cryopreserved mononuclear cells from lymph nodes or periphery were thawed and washed in R10 media containing RPMI with $10 \%$ fetal bovine calf serum, $1 \%$ HEPES, and L-glutamine plus penicillin/ streptomycin $(2 \mathrm{mM}, 100 \mathrm{U} / \mathrm{ml}, 100 \mu \mathrm{g} / \mathrm{ml}$, respectively). To assess HIVspecific responses, thawed and rested cells were stimulated with GP120 or 
Table 1

Characteristics of the study participants

\begin{tabular}{|c|c|c|c|c|c|c|}
\hline IDA & LNs & PBMCs & Cohort & Viral load (copies/ml) & CD4 count (cells/ $\mu \mathrm{l})$ & CD8 count (cells/ul) \\
\hline 1112 & Yes & Yes & Chronic treated & 5,000 & 573 & 1,304 \\
\hline 1102 & Yes & Yes & Chronic treated & 49 & 555 & - \\
\hline 1105 & Yes & & Chronic treated & 49 & 259 & 396 \\
\hline 1109 & Yes & Yes & Chronic treated & 24 & 731 & 651 \\
\hline 1111 & Yes & Yes & Chronic treated & 49 & 282 & 801 \\
\hline 1110 & Yes & Yes & Chronic treated & 49 & 176 & 640 \\
\hline 1101 & Yes & Yes & Chronic treated & 49 & 691 & 690 \\
\hline 1107 & Yes & Yes & Chronic treated & - & - & - \\
\hline 1119 & Yes & Yes & Chronic treated & 6 & 874 & 1,199 \\
\hline 1131 & Yes & & Chronic treated & 9 & 653 & 836 \\
\hline Average & & & & 587 & 533 & 815 \\
\hline 1103 & Yes & Yes & Chronic untreated & 34,000 & 296 & 814 \\
\hline 1104 & Yes & Yes & Chronic untreated & 43,000 & 514 & 1,007 \\
\hline 1106 & Yes & Yes & Chronic untreated & 9,500 & 459 & 851 \\
\hline 1108 & Yes & Yes & Chronic untreated & 250,000 & - & - \\
\hline 1113 & Yes & Yes & Chronic untreated & 36,000 & 306 & 686 \\
\hline 1114 & Yes & Yes & Chronic untreated & 32,000 & 588 & 958 \\
\hline 1115 & Yes & & Chronic untreated & 73,500 & 444 & - \\
\hline 1116 & Yes & & Chronic untreated & 20,500 & 401 & 1,293 \\
\hline 1117 & Yes & & Chronic untreated & 10,400 & 391 & 393 \\
\hline 1118 & Yes & & Chronic untreated & 704,500 & 639 & - \\
\hline 1119 & Yes & Yes & Chronic untreated & 22,500 & 465 & 1,245 \\
\hline 1120 & Yes & & Chronic untreated & 115,500 & 699 & 1,786 \\
\hline 1128 & Yes & & Chronic untreated & 145,500 & 607 & 419 \\
\hline 1129 & Yes & & Chronic untreated & 406,500 & 350 & 1,490 \\
\hline 1130 & Yes & & Chronic untreated & 26,500 & 313 & 499 \\
\hline 1131 & Yes & & Chronic untreated & 12,500 & 435 & 911 \\
\hline Average & & & & 121,400 & 460 & 950 \\
\hline 1121 & Yes & & HIV $^{-}$ & NA & NA & NA \\
\hline 1122 & Yes & & HIV $^{-}$ & NA & NA & NA \\
\hline 1123 & Yes & & HIV $^{-}$ & NA & NA & NA \\
\hline 1124 & Yes & & HIV $^{-}$ & NA & NA & NA \\
\hline 1125 & Yes & & HIV $^{-}$ & NA & NA & NA \\
\hline 1126 & Yes & & HIV- $^{-}$ & NA & NA & NA \\
\hline 1127 & Yes & & HIV $^{-}$ & NA & NA & NA \\
\hline
\end{tabular}

Cells were tested in the lymph nodes (LNs) and PBMCs of the indicated study participants. AID numbers were assigned randomly by a researcher. -, not done.

GAG overlapping peptide pools from consensus clade B sequence $(2 \mu \mathrm{g} / \mathrm{ml})$ in the presence of the costimulatory molecules CD28/CD49d $(1 \mu \mathrm{l} / \mathrm{ml})$ (BD FastImmune), as previously described (49). As a positive control, cells were stimulated with a combination of PMA $(0.1 \mu \mathrm{g} / \mathrm{ml})$ and ionomycin $(0.05 \mu \mathrm{g} / \mathrm{ml})$ (Supplemental Figure 2). Fifteen minutes into the stimulation, brefeldin A and monensin $(0.5 \mu \mathrm{l} / \mathrm{ml})$ (BD Pharmingen) were added. Samples were incubated at $37^{\circ} \mathrm{C}$ and $5 \% \mathrm{CO}_{2}$ in R10 for a total of 6 hours.

Multiparameter flow cytometric staining. Following stimulation, cells were washed with phosphate-buffered saline and stained with amine-reactive viability dye (LIVE/DEAD Blue, Invitrogen). Cells were then surface stained at room temperature for 15 minutes using the following antibodies: CD3Pacific Blue (UCHT1) and CD4-Qdot705 (S3.5) (both from Invitrogen); CD14-Pacific Blue (M5E2), CD16-Pacific Blue (3G8), CXCR5-AF488 (RF8B2), CD19-APC-Cy7 (SJ25C1), CD38-AF700 (HIT2), IgD-PE (IA6-2), IgM-FITC (G20-127), and CD27-APC (M-T271) (all from BD Biosciences); CCR7-AF647 (TG8/CCR7), PD-1-Brilliant Violet 421 (EH12.2H7), and CD45RA-APC-Cy7 (HI100) (all from Biolegend). Intracellular staining was performed at room temperature for 30 minutes following fixation and permeabilization with FIX/PERM buffers (Invitrogen) using the following antibodies: CD3-Qdot605 (UCHT1, Invitrogen), BCL6-PE (K112-91, BD Pharmigen), IFN $\gamma$-PE-Cy7 (B27, Biolegend), IL-21-AF647 (3A3-N2.1, BD Pharmigen), and TNF- $\alpha$-AF700 (MAb11, BD Pharmigen) (50).

Flow cytometric analysis. Samples were acquired on a 5-laser LSR Fortessa (BD Biosciences) multicolor flow cytometer. Compensation was performed using compensation beads labeled with a single antibody (CompBeads, BD Biosciences) and amine-dye reactive beads ( $\mathrm{ArC}$, Invitrogen). Cytometer settings were standardized and tracked between runs using multi-fluorescent calibration beads (Rainbow Fluorescent Particles, Spherotech). Data were analyzed using FlowJo version 9.2 (TreeStar). Lymphocytes were defined by FSC/SSC, and doublets were excluded by FSC-A/FSC-H. Live cells were gated as negatives for Blue Live/Dead staining, and CD4 $\mathrm{T}$ cells were defined as being $\mathrm{CD}^{+} \mathrm{CD}^{-}$ $\mathrm{CD}^{+}$(gating strategy, Supplemental Figure 2). T cell memory phenotype was characterized by CCR7 and CD45RA expression, where naive cells were defined as $\mathrm{CCR}^{+}{ }^{+} \mathrm{CD} 45 \mathrm{RA}^{+}$, central memory cells were defined as $\mathrm{CCR} 7^{+} \mathrm{CD} 45 \mathrm{RA}{ }^{-}$, TEMRA cells were defined as CCR7-CD45RA ${ }^{+}$, and effector memory cells were defined as $\mathrm{CCR} 7^{+} \mathrm{CD} 45 \mathrm{RA}^{-}$. B cells were defined as $\mathrm{CD}^{-} \mathrm{CD} 14^{-} \mathrm{CD} 16^{-}$ $\mathrm{CD}^{+} 9^{+}$(Supplemental Figure 1). B cells were further characterized as distinct subsets based on $\mathrm{CD} 38$ and $\mathrm{IgD}$ expression as follows: $\mathrm{Bm} 1, \mathrm{CD} 38-\operatorname{IgD}{ }^{+} ; \mathrm{Bm} 2$, 
$\mathrm{CD}^{+} 8^{+} \mathrm{IgD}^{+} ; \mathrm{Bm}^{\prime}$, $\mathrm{CD}^{2} 8^{++} \mathrm{IgD}^{+} ; \mathrm{Bm} 3 / 4, \mathrm{CD}^{2} 8^{++} \mathrm{IgD}^{-}$; $\mathrm{Bm} 5$ early, $\mathrm{CD}^{2} 8^{+} \mathrm{IgD}^{-}$; $\mathrm{Bm} 5$ late, $\mathrm{CD} 38^{-} \mathrm{IgD}^{-}$; and plasma cells, $\mathrm{CD}^{2} 8^{++} \mathrm{IgD}^{-}$. Immature transitional $\mathrm{B}$ cells were defined as $\mathrm{CD} 19^{+} \mathrm{CD} 27^{-} \mathrm{CD} 38^{++} \mathrm{IgM}^{+} \mathrm{IgD}^{+}$. All HIV-specific T cell responses were background subtracted based on negative controls.

Measurement of antibody levels. The level of IgG subclasses, IgG1, IgG2, IgG3, and IgG4, was measured in sera of HIV-positive individuals by a clinical standardized nephelometric assay on a Behring Nephelometer Analyzer (BD Biosciences), as previously described (51). Total IgG was calculated as the sum of the 4 subclasses, which has been shown to give high accuracy, using nephelometric assay (51).

Statistics. Statistical analysis was performed using GraphPad Prism 5.0 (GraphPad Software). For parameter comparison between subject groups, a Mann-Whitney $U$ test was used. Post-hoc test following Kruskal-Wallis was used for the comparison of multiple groups. Data in dot plots represents mean \pm SEM. Spearman's rank test was used for correlations. $P$ values of less than 0.05 were considered significant.

Study approval. The institutional review boards of the University Medical Center Eppendorf and Massachusetts General Hospital approved this study, and each participant had provided written, informed consent for participation in the study.

\section{Acknowledgments}

We thank all individuals whose participation enabled this study. This study was funded by the NIH (1R01-AI091450-01 and 1R01AI094602-01). P. Hartjen and J. Schulze zur Wiesch were supported by the German Ministry for Education and Research and the intramural funding program of the University Medical Center Eppendorf, the Werner-Otto foundation, Deutsches Zentrum für Infektionsforschung, and the German Research Agency (SFB841 project A6) (to J. Schulze zur Wiesch).

Received for publication April 13, 2012, and accepted in revised form July 5, 2012.

Address correspondence to: Hendrik Streeck, US Military HIV Research Program, 6720-A Rockledge Drive, Bethesda, Maryland 20817, USA. Phone: 301.500.3600; Fax: 301.500.3666; E-mail: hstreeck@hivresearch.org.

Hendrik Streeck's present address is: US Military HIV Research Program, Bethesda, Maryland 20817, USA.
1. Moir S, Fauci AS. B cells in HIV infection and disease. Nat Rev Immunol. 2009;9(4):235-245.

2. Titanji K, et al. Primary HIV-1 infection sets the stage for important B lymphocyte dysfunctions. AIDS. 2005;19(17):1947-1955.

3. Malaspina A, et al. Appearance of immature/transitional B cells in HIV-infected individuals with advanced disease: correlation with increased IL-7. Proc Natl Acad Sci U S A. 2006;103(7):2262-2267.

4. De Milito A, et al. Mechanisms of hypergammaglobulinemia and impaired antigen-specific humoral immunity in HIV-1 infection. Blood. 2004;103(6):2180-2186.

5. Schnittman SM, Lane HC, Higgins SE, Folks T, Fauci AS. Direct polyclonal activation of human B lymphocytes by the acquired immune deficiency syndrome virus. Science. 1986;233(4768):1084-1086.

6. Lefevre EA, Krzysiek R, Loret EP, Galanaud P, Richard Y. Cutting edge: HIV-1 Tat protein differentially modulates the B cell response of naive, memory, and germinal center B cells. J Immunol. 1999;163(3):1119-1122.

7. Qiao X, He B, Chiu A, Knowles DM, Chadburn A, Cerutti A. Human immunodeficiency virus $1 \mathrm{Nef}$ suppresses CD40-dependent immunoglobulin class switching in bystander B cells. Nat Immunol. 2006;7(3):302-310.

8. Popovic M, et al. Persistence of HIV-1 structural proteins and glycoproteins in lymph nodes of patients under highly active antiretroviral therapy. Proc Natl Acad Sci US A. 2005;102(41):14807-14812.

9. Moir S, et al. B cells of HIV-1-infected patients bind virions through $\mathrm{CD} 21$-complement interactions and transmit infectious virus to activated $\mathrm{T}$ cells. J Exp Med. 2000;192(5):637-646.

10. Morris L, et al. HIV-1 antigen-specific and-nonspecific $B$ cell responses are sensitive to combination antiretroviral therapy. J Exp Med. 1998;188(2):233-245.

11. Mattapallil JJ, Douek DC, Hill B, Nishimura Y, Martin $\mathrm{M}$, Roederer M. Massive infection and loss of memory CD4+ T cells in multiple tissues during acute SIV infection. Nature. 2005;434(7037):1093-1097.

12. McHeyzer-Williams M, Okitsu S, Wang N, McHeyzer-Williams L. Molecular programming of B cell memory. Nat Rev Immunol. 2012;12(1):24-34.

13. Crotty S. Follicular helper CD4 T cells (TFH). Annu Rev Immunol. 2011;29:621-663.

14. Yu D, et al. The transcriptional repressor Bcl-6 directs $\mathrm{T}$ follicular helper cell lineage commitment. Immunity. 2009;31(3):457-468.

15. Baumjohann D, Okada T, Ansel KM. Cutting
Edge: Distinct waves of BCL6 expression during $\mathrm{T}$ follicular helper cell development. J Immunol. 2011;187(5):2089-2092.

16. Simpson N, et al. Expansion of circulating $T$ cells resembling follicular helper $\mathrm{T}$ cells is a fixed phenotype that identifies a subset of severe systemic lupus erythematosus. Arthritis Rheum. 2010; 62(1):234-244.

17. Kroenke MA, et al. Bcl6 and Maf cooperate to instruct human follicular helper CD4 T cell differentiation. J Immunol. 2012;188(8):3734-3744.

18. Chtanova $\mathrm{T}$, et al. $\mathrm{T}$ follicular helper cells express a distinctive transcriptional profile, reflecting their role as non-Th1/Th2 effector cells that provide help for B cells. J Immunol. 2004;173(1):68-78.

19. Kuchen S, et al. Essential role of IL-21 in B cell activation, expansion, and plasma cell generation during CD4+ T cell-B cell collaboration. J Immunol. 2007;179(9):5886-5896.

20. Bussmann BM, Reiche S, Bieniek B, Krznaric I, Ackermann F, Jassoy C. Loss of HIV-specific memory B-cells as a potential mechanism for the dysfunction of the humoral immune response against HIV. Virology. 2010;397(1):7-13.

21. Haase AT. Perils at mucosal front lines for HIV and SIV and their hosts. Nat Rev Immunol. 2005;5(10):783-792.

22. Tenner-Racz K, et al. The unenlarged lymph nodes of HIV-1-infected, asymptomatic patients with high CD4 T cell counts are sites for virus replication and CD4 T cell proliferation. J Exp Med. 1998;187(6):949-959.

23. Sette A, et al. Selective CD4+ T cell help for antibody responses to a large viral pathogen: deterministic linkage of specificities. Immunity. 2008; 28(6):847-858.

24. Klasse PJ, Sanders RW, Cerutti A, Moore JP. How can HIV-type-1-Env immunogenicity be improved to facilitate antibody-based vaccine development? AIDS Res Hum Retroviruses. 2012;28(1):1-15.

25. Binley JM, et al. Differential regulation of the antibody responses to Gag and Env proteins of human immunodeficiency virus type 1 . J Virol. 1997;71(4):2799-2809.

26. Li Y, et al. Analysis of neutralization specificities in polyclonal sera derived from human immunodeficiency virus type 1 -infected individuals. J Virol. 2009;83(2):1045-1059.

27. Binley JM, et al. Profiling the specificity of neutralizing antibodies in a large panel of plasmas from patients chronically infected with human immunodeficiency virus type 1 subtypes B and C. J Virol. 2008;82(23):11651-11668.

28. Moore PL, Gray ES, Morris L. Specificity of the autologous neutralizing antibody response. Curr Opin HIV AIDS. 2009;4(5):358-363.

29. Muller F, Froland SS, Brandtzaeg P. Altered IgGsubclass distribution in lymph node cells and serum of adults infected with human immunodeficiency virus (HIV). Clin Exp Immunol. 1989;78(2):153-158.

30. Aucouturier P, et al. Serum immunoglobulin G subclass dysbalances in the lymphadenopathy syndrome and acquired immune deficiency syndrome. Clin Exp Immunol. 1986;63(1):234-240.

31. Pascual V, Liu YJ, Magalski A, de Bouteiller O, Banchereau J, Capra JD. Analysis of somatic mutation in five B cell subsets of human tonsil. J Exp Med. 1994;180(1):329-339.

32. Cappione A $3 \mathrm{rd}$, et al. Germinal center exclusion of autoreactive B cells is defective in human systemic lupus erythematosus. J Clin Invest. 2005;115(11):3205-3216.

33. Fahey LM, Wilson EB, Elsaesser H, Fistonich CD, McGavern DB, Brooks DG. Viral persistence redirects CD4 $\mathrm{T}$ cell differentiation toward $\mathrm{T}$ follicular helper cells. J Exp Med. 2011;208(5):987-999.

34. Fazilleau N, McHeyzer-Williams LJ, Rosen H, McHeyzer-Williams MG. The function of follicular helper $\mathrm{T}$ cells is regulated by the strength of $\mathrm{T}$ cell antigen receptor binding. Nat Immunol. 2009;10(4):375-384.

35. Douek DC, et al. HIV preferentially infects HIV-specific CD4+ T cells. Nature. 2002;417(6884):95-98.

36. Petrovas C, et al. CD4 T follicular helper cell dynamics during SIV infection. J Clin Invest. 2012;122(9):3281-3294.

37. Ozaki K, et al. Regulation of B cell differentiation and plasma cell generation by IL-21, a novel inducer of Blimp-1 and Bcl-6. J Immunol. 2004; 173(9):5361-5371.

38. Linterman MA, et al. IL-21 acts directly on B cells to regulate Bcl-6 expression and germinal center responses. J Exp Med. 2010;207(2):353-363.

39. Linterman MA, et al. Foxp $3+$ follicular regulatory $T$ cells control the germinal center response. Nat Med. 2011;17(8):975-982.

40. Chung Y, et al. Follicular regulatory T cells expressing Foxp3 and Bcl-6 suppress germinal center reactions. Nat Med. 2011;17(8):983-988.

41. Malaspina A, et al. Idiopathic CD4+ T lymphocytopenia is associated with increases in immature/ 
transitional B cells and serum levels of IL-7. Blood. 2007;109(5):2086-2088.

42. Ettinger R, et al. IL-2 1 induces differentiation of human naive and memory $B$ cells into antibody-secreting plasma cells. J Immunol. 2005; 175(12):7867-7879.

43. Bryant VL, et al. Cytokine-mediated regulation of human B cell differentiation into Ig-secreting cells: predominant role of IL-21 produced by CXCR5+ T follicular helper cells. J Immunol. 2007;179(12):8180-8190.

44. Shacklett BL. Mucosal immunity to HIV: a review of recent literature. Curr Opin HIV AIDS. 2008;3(5):541-547.
45. Brenchley JM, et al. CD4+ T cell depletion during all stages of HIV disease occurs predominantly in the gastrointestinal tract. J Exp Med. 2004; 200(6):749-759.

46. Doria-Rose NA, et al. Breadth of human immunodeficiency virus-specific neutralizing activity in sera: clustering analysis and association with clinical variables. J Virol. 2010;84(3):1631-1636.

47. Zhou T, et al. Structural basis for broad and potent neutralization of HIV-1 by antibody VRC01. Science. 2010;329(5993):811-817.

48. Pancera M, et al. Crystal structure of PG16 and chimeric dissection with somatically related PG9: structure-function analysis of two quaternary-spe- cific antibodies that effectively neutralize HIV-1. JVirol. 2010;84(16):8098-8110.

49. Soghoian DZ, et al. HIV-specific cytolytic CD4 $\mathrm{T}$ cell responses during acute HIV infection predict disease outcome. Sci Transl Med. 2012; 4(123):123ra125.

50. Chevalier MF, et al. HIV-1-specific interleukin-21+ $\mathrm{CD} 4+\mathrm{T}$ cell responses contribute to durable viral control through the modulation of HIV-specific CD8+ T cell function. J Virol. 2011;85(2):733-741.

51. Vlug A, Nieuwenhuys EJ, van Eijk RV, Geertzen HG, van Houte AJ. Nephelometric measurements of human IgG subclasses and their reference ranges. Ann Biol Clin (Paris). 1994;52(7-8):561-567. 